\title{
Generating Theorems for Charged Anisotropy in General Relativity
}

\author{
Napasorn Jongjittanon ${ }^{1,}$, , Petarpa Boonserm², Tritos Ngampitipan ${ }^{3}$ \\ ${ }^{1}$ Department of Physics, Faculty of Science, Chulalongkorn University, Bangkok, Thailand \\ ${ }^{2}$ Department of Mathematics and Computer Science, Faculty of Science, Chulalongkorn University, Bangkok, Thailand \\ ${ }^{3}$ Faculty of Science, Chandrakasem Rajabhat University, Bangkok, Thailand
}

Email address:

njongjittanon@gmail.com (N. Jongjittanon), Petarpa.Boonserm@gmail.com (P. Boonserm), tritos.ngampitipan@gmail.com (T. Ngampitipan) ${ }^{*}$ Corresponding author

\section{To cite this article:}

Napasorn Jongjittanon, Petarpa Boonserm, Tritos Ngampitipan. Generating Theorems for Charged Anisotropy in General Relativity. American Journal of Physics and Applications. Vol. 4, No. 2, 2016, pp. 50-56. doi: 10.11648/j.ajpa.20160402.13

Received: February 26, 2016; Accepted: March 18, 2016; Published: March 25, 2016

\begin{abstract}
For describing the interior of a spherical object in the general relativistic frame, some objects can be considered using the concept of perfect fluid spheres for simplicity. The absence of heat conduction and shear stress, and the presence of isotropic pressure are the characteristics of perfect fluid spheres. Previous works in this field constitute finding solutions for perfect fluid spheres in various coordinates. In this work, we are interested in generating anisotropic solution for fluid spheres. The particular property of anisotropy, which differs from the property of perfect fluid spheres, is that the radial pressure and the transverse pressure are not equal. One cause of anisotropy is the presence of charge inside an object. Anisotropic fluid spheres are models for describing a charged star such as a neutron star. An important tool in studying fluid sphere solutions is the solution generating algorithm. This technique can be used to generate new solution from known solutions without having to solve Einstein's equation directly. The solution generating theorems for anisotropic fluid spheres are constructed in terms of the metric of spacetime. The other purpose is to classify the types of solution into seed and non-seed metrices.
\end{abstract}

Keywords: General Relativity, Charged Anisotropy, Fluid Spheres

\section{Introduction}

General theory of relativity was first published in 1915 , which introduced a completely new perspective in the understanding of physics. The theory presents new ideas about gravity. In classical physics, gravity is a force exerted by a mass, which attracts another mass in certain proximity. If the mass disappears, the force is suddenly lost. However, according to the general relativity, gravity is not a force. It is a consequence of a mass on spacetime. The spacetime within which the matter sits will be curved because of the presence of mass. This curvature is recognized as gravity. The other mass in proximity is also aware of the presence of gravity in spacetime. Anything that travels through spacetime has its speed limit not exceeding the speed of light. If the mass suddenly disappears, the other mass has to take the time to realize the missing gravity. The center of the theory is the Einstein's field equations which express the relationship between mass (or energy) and the geometry of spacetime,

$$
G_{\mu \nu}=8 \pi T_{\mu \nu}
$$

The right hand side of the equation describes mass and energy, while the left hand side is the spacetime part of the equation. Einstein's field equations are nonlinear differential equation with coupling variables. It is hard to solve the equation directly without any assumption or symmetry. The first physicist who solved for the exact solution was Karl Schwarzschild. He applied spherical symmetry to Einstein's theory to explain the gravitational field produced by some objects in a model of fluid spheres [1]. After that, perfect fluid spheres became known as the first approximation in the construction of a realistic model for a general relativistic star [2-17].

We assume that all matter in a star can be equated to perfect fluid sphere, allowing for symmetry that makes Einstein's field equations simpler. Perfect fluid spheres can represent a spherically symmetric ideal object which is filled by mass or 
energy. The definitions for perfect fluid in this work differ from fluid mechanics. They are expressed in terms of energy-momentum-stress tensor $T^{\mu \nu}$;

$$
T_{\mu \nu}=\left[\begin{array}{llll}
T_{00} & T_{01} & T_{02} & T_{03} \\
T_{10} & T_{11} & T_{12} & T_{13} \\
T_{20} & T_{21} & T_{22} & T_{23} \\
T_{30} & T_{31} & T_{32} & T_{33}
\end{array}\right] .
$$

The quantity $T^{\mu \nu}$ describes four momentum $p^{\mu}$ across the surface constant $x^{v}$ [3].

In general relativity, the three properties associated with perfect fluid are: no shear stress, no heat (energy) conduction, and isotropic pressure. In Veilbein formalism, these properties direct the energy-momentum-stress tensor of perfect fluid to the following diagonal matrix;

$$
T_{\hat{a} \hat{b}}=\left[\begin{array}{cccc}
\rho & 0 & 0 & 0 \\
0 & p & 0 & 0 \\
0 & 0 & p & 0 \\
0 & 0 & 0 & p
\end{array}\right] .
$$

For the isotropic property, the pressure in every direction must be equal. Using equation (1), we obtain Einstein tensor $G_{\mu \nu}$ for perfect fluid spheres; and perfect fluid constraints $G_{\hat{r} \hat{r}}=G_{\widehat{\theta} \hat{\theta}}=G_{\widehat{\phi} \hat{\phi}}$. The hat symbols above the coordinates are used to represent the quantities in Veilbein formalism. This form has a non-coordinate basis and agrees with the observer's view [2, 9-10, 15-17].

\section{Solution Generating Theorems for Charged Anisotropy}

\subsection{Anisotropy for a Spherical Symmetry Object}

Many solutions of Einstein's field equations with perfect fluid constraints were explored in various coordinate systems [4-5]. Each solution explains different systems in different conditions. Recently, some algorithmic techniques have been developed to generate new solutions from known perfect fluid spheres [2]. This is very interesting because we no longer need to solve Einstein's field equations directly to obtain the solutions. This concept also classifies the type of metric solution and exhibits the association of the solutions in distinct conditions.

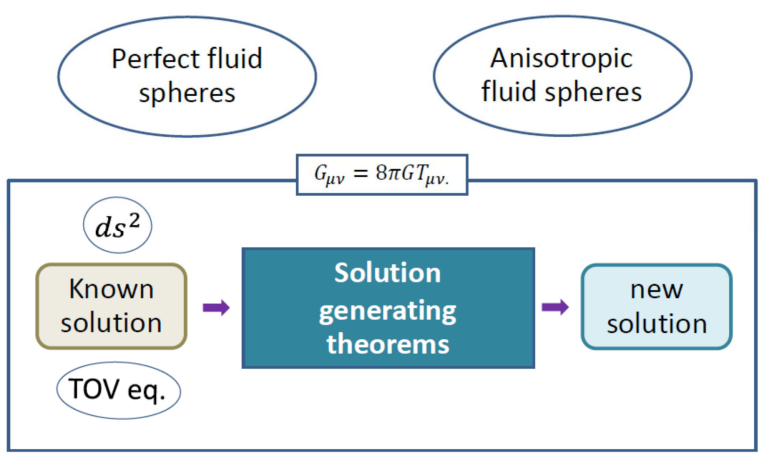

Figure 1. This figure shows the working of solution generating theorem [2, 9].
For finding solution generating theorems, a metric (or line element) of an object was written in suitable coordinate. By definition of the geometric element of Einstein's field equation, $G_{\mu \nu} \equiv R_{\mu \nu}-\frac{1}{2} g_{\mu \nu} R$, the Einstein tensor $G_{\mu \nu}$ can be obtained from the metric. We can set the perfect fluid constraints to $G_{\mu \nu}$ for ordinary differential equation, which can be used to build solution generating theorems. These theorems deform perfect fluid spheres in terms of spacetime geometry.

In case of spherical objects with a charge on the inside, the electromagnetic charge makes the objects anisotropic. The radial pressure and the transverse pressure in these kinds of objects are unequal. The energy-momentum-stress tensor for anisotropy is

$$
T_{\hat{a} \hat{b}}=\left[\begin{array}{cccc}
\rho & 0 & 0 & 0 \\
0 & p_{r} & 0 & 0 \\
0 & 0 & p_{t} & 0 \\
0 & 0 & 0 & p_{t}
\end{array}\right] .
$$

The constraint of perfect fluid spheres cannot be used in constructing solution generating theorems. Therefore, we have to set a new ordinary differential equation that satisfies anisotropic fluid spheres from Einstein's equation. This equation gives an Einstein tensor that agrees with the anisotropic energy momentum stress tensor. This model is a primary model that can be applied to a real object in our universe; the neutron stars [2-17].

The type of compact star that a star will eventually become, in the end point, is up to the mass of the star. A neutron star is one type of an end point star. It is the result of gravitational collapse of a star into a very small size compared to the mass. The matter of the star is condensed and any chemical potential becomes broken up. Finally, there are only neutrons and elementary particles that remain in the star [18-19].

In studying neutron stars, the first object we can observe is pulsars, which is created from the rapid rotation of neutron stars. Pulsars are the key evidence of magnetic fields in the stars. Additionally, the large magnetic fields that can be observed indicate that the magnetic fields in a star are anisotropic [8]. Moreover, we also found that the matters of a neutron star constitute several particle types with different charges. The lightest charged particles, electrons, pass into the boundary and create an unbalanced electric field on the surface of the star. The electric field affects the energy-momentum-stress tensor, the tensor that was filled with four-momentum across the surface, which gives us the anisotropic pressure. A neutron star is an example of an anisotropic object that we are interested in for this study.

\subsection{Solution Generating Theorems for Charged Anisotropy}

\subsubsection{Ordinary Differential Equation for Charged Anisotropy}

Suppose the solution of charged fluid spheres were defined as a specific metric [2-10, 17]

$$
d s^{2}=-\zeta_{0}(r)^{2} d t^{2}+\frac{d r^{2}}{B_{0}(r)}+r^{2} d \Omega^{2}
$$


or with the notation $\left\{\zeta_{0}, B_{0}\right\}$.

From Einstein's field equation, we obtain the Einstein tensor [18-19]

$$
\begin{gathered}
G_{\hat{r} \hat{r}}=\frac{4 B \zeta^{\prime} r-2 \zeta+2 \zeta B}{2 r^{2} \zeta}, \\
G_{\widehat{\theta} \hat{\theta}}=\frac{B^{\prime} \zeta r+2 B \zeta^{\prime} r+2 r^{2} \zeta^{\prime \prime} B+r^{2} \zeta^{\prime} B^{\prime}}{2 r^{2} \zeta} .
\end{gathered}
$$

Then setting $G_{\hat{r} \hat{r}}-G_{\hat{\theta} \hat{\theta}}=8 \pi \Delta$, we obtain a linear homogeneous second order ODE for $\zeta(r)$

$$
\begin{gathered}
2 r^{2} B \zeta^{\prime \prime}+\left(r^{2} B^{\prime}-2 B r\right) \zeta^{\prime} \\
+\left(2-2 B+B^{\prime} r+16 \pi \Delta r^{2}\right) \zeta=0,
\end{gathered}
$$

which can be rearranged to a first order linear non-homogeneous equation in $B(r)$

$$
\begin{gathered}
\left(r^{2} \zeta^{\prime}+r \zeta\right) B^{\prime}+\left(2 r^{2} \zeta^{\prime \prime}-2 r \zeta^{\prime}-2 \zeta\right) B \\
+\left(2+16 \pi \Delta r^{2}\right) \zeta=0 .
\end{gathered}
$$

Theorem 1. Suppose $\left\{\zeta_{0}(r), B_{0}(r)\right\}$ represents a charged fluid sphere. Define

$$
\Delta_{0}=\left(\frac{\zeta_{0}(r)}{\zeta_{0}(r)+r \zeta_{0}^{\prime}}\right)^{2} r^{2} \exp \left\{2 \int \frac{\zeta_{0}^{\prime}(r)}{\zeta_{0}(r)} \frac{\zeta_{0}(r)-r \zeta_{0}^{\prime}(r)}{\zeta_{0}(r)+r \zeta_{0}^{\prime}(r)} d r\right\} .
$$

Then for all $\lambda$, the geometry defined by holding $\zeta_{0}(r)$ fixed and setting

$$
d s^{2}=-\zeta_{0}(r)^{2} d t^{2}+\frac{d r^{2}}{B_{0}(r)+\lambda \Delta_{0}(r)}+r^{2} d \Omega^{2}
$$

is also a charged fluid sphere. That is, the mapping of

$$
T_{1}(\lambda):\left\{\zeta_{0}, B_{0}\right\} \mapsto\left\{\zeta_{0}, B_{0}+\lambda \Delta_{0}\left(\zeta_{0}\right)\right\}
$$

takes charged fluid spheres into charged fluid spheres. Furthermore, a second application of the transformation does not yield a new information; $T_{1}$ is "idempotent", in the sense that

$$
\begin{gathered}
T_{1}\left(\lambda_{n}\right) \circ \ldots \circ T_{1}\left(\lambda_{2}\right) \circ T_{1}\left(\lambda_{1}\right): \\
\left\{\zeta_{0}, B_{0}\right\} \mapsto\left\{\zeta_{0}, B_{0}+\left(\sum_{i=1}^{n} \lambda_{i}\right) \Delta_{0}\left(\zeta_{0}\right)\right\} .
\end{gathered}
$$

For a general form

$$
\prod_{i=1}^{n} T_{1} \triangleq T_{1}
$$

the symbol $\triangleq$ represents "equality up to relabeling of the parameters"[2].

Proof for theorem 1. Assume the metric represents a charged fluid sphere

$$
d s^{2}=-\zeta_{0}(r)^{2} d t^{2}+\frac{d r^{2}}{B_{0}(r)}+r^{2} d \Omega^{2} .
$$

We know $\zeta_{0}$ and $B_{0}$ are solutions of charged fluid sphere. Therefore, they satisfy ODE (8)

$$
\left(r^{2} \zeta^{\prime}+r \zeta\right) B^{\prime}+\left(2 r^{2} \zeta^{\prime \prime}-2 r \zeta^{\prime}-2 \zeta\right) B+\left(2+16 \pi \Delta r^{2}\right) \zeta_{0}=0 .
$$

If we modified the metric as

$$
d s^{2}=-\zeta_{0}(r)^{2} d t^{2}+\frac{d r^{2}}{B_{1}(r)}+r^{2} d \Omega^{2},
$$

then $B_{1}(r)=B_{0}(r)+\lambda \Delta_{0}(r)$. For this metric, $\zeta_{0}$ and $B_{1}$ also satisfy charged fluid spheres

$$
\begin{gathered}
\left(r^{2} \zeta_{0}{ }^{\prime}+r \zeta_{0}\right) B_{1}{ }^{\prime}+\left(2 r^{2} \zeta_{0}{ }^{\prime \prime}-2 r \zeta_{0}{ }^{\prime}-2 \zeta_{0}\right) B_{1} \\
+\left(2+16 \pi \Delta r^{2}\right) \zeta_{0}=0 .
\end{gathered}
$$

Substituting $B_{1}(r)=B_{0}(r)+\lambda \Delta_{0}(r)$ into equation (16)

$$
\begin{gathered}
\left(r^{2} \zeta_{0}{ }^{\prime}+r \zeta_{0}\right) B_{0}{ }^{\prime}+\left(2 r^{2} \zeta_{0}{ }^{\prime \prime}-2 r \zeta_{0}{ }^{\prime}-2 \zeta_{0}\right) B_{0} \\
+\left(2 r^{2} \zeta_{0}{ }^{\prime \prime}-2 r \zeta_{0}{ }^{\prime}-2 \zeta_{0}\right) B_{0} \\
+\left(2 r^{2} \zeta_{0}{ }^{\prime \prime}-2 r \zeta_{0}{ }^{\prime}-2 \zeta_{0}\right) \lambda \Delta_{0}+\left(2+16 \pi \Delta r^{2}\right) \zeta_{0}=0 .
\end{gathered}
$$

Using equation (8) gives

$$
\left(r^{2} \zeta_{0}{ }^{\prime}+r \zeta_{0}\right) \lambda \Delta_{0}^{\prime}+\left(2 r^{2} \zeta_{0}{ }^{\prime \prime}-2 r \zeta_{0}{ }^{\prime}-2 \zeta_{0}\right) \lambda \Delta_{0}=0 .
$$

This homogeneous first order differential equation in $\Delta_{0}$ leads to

Proof for idempotent property. For a second application of theorem 1, the mapping is

$$
T_{1}\left(\lambda_{2}\right) \circ T_{1}\left(\lambda_{1}\right):\left\{\zeta_{0}, B_{0}\right\} \mapsto\left\{\zeta_{0}, B_{2}\right\},
$$

where $B_{2}=B_{1}+\lambda_{2} \Delta_{0}\left(\zeta_{0}\right)$ and $B_{1}=B_{0}+\lambda_{1} \Delta_{0}\left(\zeta_{0}\right)$. So,

$$
T_{1}\left(\lambda_{2}\right) \circ T_{1}\left(\lambda_{1}\right):\left\{\zeta_{0}, B_{0}\right\} \mapsto\left\{\zeta_{0}, B_{0}+\left(\lambda_{2}+\lambda_{1}\right) \Delta_{0}\right\} .
$$

For $\mathrm{n}$ application of theorem 1, the mapping is

$$
\begin{gathered}
T_{1}\left(\lambda_{n}\right) \circ \ldots \circ T_{1}\left(\lambda_{2}\right) \circ T_{1}\left(\lambda_{1}\right): \\
\left\{\zeta_{0}, B_{0}\right\} \mapsto\left\{\zeta_{0}, B_{0}+\left(\sum_{i=1}^{n} \lambda_{i}\right) \Delta_{0}\left(\zeta_{0}\right)\right\} .
\end{gathered}
$$

Corollary 1. Let $\left\{\zeta_{0}, B_{a}\right\}$ and $\left\{\zeta_{0}, B_{b}\right\}$ both represent charged fluid spheres, then for all $\mathrm{p}$

$$
\left\{\zeta_{0}, p B_{a}+(1-p) B_{b}\right\}
$$

is also a charged fluid sphere. Furthermore, all fluid spheres for a fixed $\zeta_{0}$ can also be written in this form.

Proof for corollary 1. Suppose $\left\{\zeta_{0}, B_{a}\right\}$ and $\left\{\zeta_{0}, B_{b}\right\}$ represent charged fluid spheres, the solutions could satisfy (8)

$$
\begin{gathered}
\left(r^{2} \zeta^{\prime}+r \zeta\right)\left(B_{a}\right)^{\prime}+\left(2 r^{2} \zeta^{\prime \prime}-2 r \zeta^{\prime}-2 \zeta\right) B_{a} \\
+\left(2+16 \pi \Delta r^{2}\right) \zeta=0, \\
\left(r^{2} \zeta^{\prime}+r \zeta\right)\left(B_{b}\right)^{\prime}+\left(2 r^{2} \zeta^{\prime \prime}-2 r \zeta^{\prime}-2 \zeta\right) B_{b} \\
+\left(2+16 \pi \Delta r^{2}\right) \zeta=0 .
\end{gathered}
$$

also represents charged fluid spheres,

$$
\begin{gathered}
+\left(2 r^{2} \zeta^{\prime \prime}-2 r \zeta^{\prime}-2 \zeta\right)\left(p B_{a}+(1-p) B_{b}\right) \\
\left(r^{2} \zeta^{\prime}+r \zeta\right)\left(p B_{a}+(1-p) B_{b}\right)^{\prime}
\end{gathered}
$$




$$
\begin{gathered}
+\left(2+16 \pi \Delta r^{2}\right) \zeta=0 . \\
\left\{\zeta_{0}, p B_{a}+(1-p) B_{b}\right\} \\
\left(r^{2} \zeta^{\prime}+r \zeta\right) p\left(B_{a}\right)^{\prime}+\left(r^{2} \zeta^{\prime}+r \zeta\right)(1-p)\left(B_{b}\right)^{\prime} \\
+\left(2 r^{2} \zeta^{\prime \prime}-2 r \zeta^{\prime}-2 \zeta\right) p B_{a} \\
+\left(2 r^{2} \zeta^{\prime \prime}-2 r \zeta^{\prime}-2 \zeta\right)(1-p) B_{b} \\
+\left(2+16 \pi \Delta r^{2}\right) \zeta=0
\end{gathered}
$$

From $(19)-\left[p^{*}(17)+(1-p) *(18)\right]$;

$$
\begin{gathered}
-p\left(2+16 \pi \Delta r^{2}\right) \zeta-(1-p)\left(2+16 \pi \Delta r^{2}\right) \zeta \\
+\left(2+16 \pi \Delta r^{2}\right) \zeta=0
\end{gathered}
$$

Therefore, $\left\{\zeta_{0}, p B_{a}+(1-p) B_{b}\right\}$ represents a charged fluid sphere.

Application of theorem 1 to the Reissner-Nordstrom metric

\begin{tabular}{|c|c|c|}
\hline $\begin{array}{c}\text { Starting metric: } \\
\text { Reissner-Nordström }\end{array}$ & $\mathrm{T}_{1}$ & $\begin{array}{c}\text { New solution: } \\
\text { equation (21.b) }\end{array}$ \\
\hline
\end{tabular}

Figure 2. This structure shows that when we apply theorem 1 to the Reissner-Nordstrom metric, we get a new solution.

The Reissner-Nordstrom metric represent static spherical spacetime with charge,

$$
\begin{gathered}
d s^{2}=-\left(1-\frac{R_{s}}{r}+\frac{R_{Q}{ }^{2}}{r^{2}}\right) d t^{2} \\
+\left(1-\frac{R_{S}}{r}+\frac{R_{Q}^{2}}{r^{2}}\right)^{-1} d r^{2}+r^{2} d \Omega^{2} .
\end{gathered}
$$

Where $R_{S}$ is the Schwarzschild radius and $R_{Q}$ is a characteristic length scale of charge. For this metric, $\zeta_{0}=$ $\sqrt{1-\frac{R_{S}}{r}+\frac{R_{Q}^{2}}{r^{2}}}$

In applying theorem 1 , use equation (9)

$$
\begin{gathered}
\Delta_{0}=\left(\frac{\zeta_{0}(r)}{\zeta_{0}(r)+r \zeta_{0}^{\prime}}\right)^{2} r^{2} \exp \left\{2 \int \frac{\zeta_{0}^{\prime}(r)}{\zeta_{0}(r)} \frac{\zeta_{0}(r)-r \zeta_{0}^{\prime}(r)}{\zeta_{0}(r)+r \zeta_{0}^{\prime}(r)} d r\right\}, \\
\Delta_{0}=\frac{r^{2}}{\left[\left(r \zeta_{0}\right)^{\prime}\right]^{2}} \exp \left\{\int \frac{4 \zeta_{0}^{\prime}}{\left(r \zeta_{0}\right)^{\prime}} d r\right\} .
\end{gathered}
$$

From calculations using the Maple programme, we obtain

$$
\Delta_{0}=\frac{r^{2}}{\left(1-\frac{R_{S}}{2}\right)^{2}} \exp \left\{\frac{8 R_{Q}^{2}}{R_{S} r}+\left(1-4 \frac{R_{Q}^{2}}{R_{S}{ }^{2}}\right) 4 \ln \left(\frac{R_{S}}{2 r}-1\right)\right\} .
$$

Then for all $\lambda$, the new metric is

$$
d s^{2}=-\zeta_{0}(r)^{2} d t^{2}+\frac{d r^{2}}{B_{0}(r)+\lambda \Delta_{0}}+r^{2} d \Omega^{2} .
$$

With this $Z_{0}$, the metric also satisfy anisotropy fluid spheres.

Theorem 2. Suppose $\left\{\zeta_{0}(r), B_{0}(r)\right\}$ represents a charged fluid sphere. Define

$$
Z_{0}(r)=\sigma+\epsilon \int \frac{r d r}{\zeta_{0}(r)^{2} \sqrt{B_{0}(r)}}
$$

Then for all $\lambda$, the geometry defined by holding $\zeta_{0}(r)$ fixed and setting

$$
d s^{2}=-\zeta_{0}(r)^{2} Z_{0}(r)^{2} d t^{2}+\frac{d r^{2}}{B_{0}(r)}+r^{2} d \Omega^{2}
$$

is also a charged fluid sphere. That is, the mapping of

$$
\left.T_{2}(\lambda):\left\{\zeta_{0}, B_{0}\right\} \mapsto\left\{\zeta_{0} Z_{0}\left(\zeta_{0}, B_{0}\right), B_{0}\right)\right\}
$$

takes charged fluid spheres into charged fluid spheres. Furthermore, a second application of the transformation does not yield a new information; $T_{2}$ is "idempotent", in the sense that $[2,10,15-17]$

$$
T_{2}\left(\sigma_{n}, \epsilon_{n}\right) \circ \ldots \circ T_{2}\left(\sigma_{2}, \epsilon_{2}\right) \circ T_{2}\left(\sigma_{1}, \epsilon_{1}\right)=T_{2}\left(\sigma_{n} \ldots \sigma_{2} \sigma_{1}, \epsilon_{n \ldots 321}\right),
$$

where

$$
\begin{aligned}
\epsilon_{n \ldots 321}=\left(\epsilon_{1} \sigma_{2} \sigma_{3}\right. & \left.\ldots \sigma_{n}\right)+\left(\sigma_{1}{ }^{-1} \epsilon_{2} \sigma_{3} \ldots \sigma_{n}\right) \\
& +\left(\sigma_{1}{ }^{-1} \sigma_{2}{ }^{-1} \epsilon_{3} \ldots \sigma_{n}\right)+\cdots \\
& +\left(\sigma_{1}{ }^{-1} \sigma_{2}{ }^{-1} \sigma_{3}{ }^{-1} \ldots \epsilon_{n}\right) .
\end{aligned}
$$

Proof for theorem 2. Assume the metric represents a charged fluid sphere

$$
d s^{2}=-\zeta_{0}(r)^{2} d t^{2}+\frac{d r^{2}}{B_{0}(r)}+r^{2} d \Omega^{2} .
$$

We know $\zeta_{0}$ and $B_{0}$ are solutions of charged fluid sphere. Therefore they satisfy ODE (7)

$$
\begin{gathered}
2 r^{2} B_{0} \zeta_{0}{ }^{\prime \prime}+\left(r^{2} B^{\prime}{ }_{0}-2 B_{0} r\right) \zeta_{0}{ }^{\prime} \\
+\left(2-2 B_{0}+B^{\prime}{ }_{0} r+16 \pi \Delta r^{2}\right) \zeta_{0}=0 .
\end{gathered}
$$

If we modified the metric as

$$
d s^{2}=-\zeta_{1}(r)^{2} d t^{2}+\frac{d r^{2}}{B_{0}(r)}+r^{2} d \Omega^{2},
$$

Then $\zeta_{1}(r)=\zeta_{0}(r) Z_{0}(r)$. For this metric, $\zeta_{1}$ and $B_{0}$ also satisfy charged fluid spheres

$$
\begin{gathered}
2 r^{2} B_{0} \zeta_{1}^{\prime \prime}+\left(r^{2} B^{\prime}{ }_{0}-2 B_{0} r\right) \zeta_{1}{ }^{\prime} \\
+\left(2-2 B_{0}+B^{\prime}{ }_{0} r+16 \pi \Delta r^{2}\right) \zeta_{1}=0 .
\end{gathered}
$$

Substituting $\zeta_{1}(r)=\zeta_{0}(r) Z_{0}(r)$ into equation (28)

$$
\begin{gathered}
{\left[2 r^{2} B_{0}\right] \zeta_{0}{ }^{\prime \prime} Z_{0}+2\left[2 r^{2} B_{0}\right] \zeta_{0}{ }^{\prime} Z^{\prime}{ }_{0}+2\left[2 r^{2} B_{0}\right] \zeta_{0} Z^{\prime \prime}{ }_{0}} \\
+\left[r^{2} B^{\prime}{ }_{0}-2 B_{0} r\right] \zeta_{0}{ }^{\prime} Z_{0}+\left[r^{2} B^{\prime}{ }_{0}-2 B_{0} r\right] \zeta_{0} Z^{\prime}{ }_{0} \\
+\left[2-2 B_{0}+B^{\prime}{ }_{0} r+16 \pi \Delta r^{2}\right] \zeta_{0} Z_{0}=0 .
\end{gathered}
$$

Using equation (7) gives

$$
\begin{gathered}
{\left[r^{2} B_{0}^{\prime}-2 B_{0} r\right] \zeta_{0} Z_{0}^{\prime}+2\left[2 r^{2} B_{0}\right] \zeta_{0} Z^{\prime}{ }_{0}} \\
+2\left[2 r^{2} B_{0}\right] \zeta_{0} Z_{0}^{\prime \prime}=0
\end{gathered}
$$


Therefore,

$$
\frac{Z_{{ }_{0}}{ }_{0}}{Z_{0}^{\prime}}=-\frac{4 r^{2} B_{0} \zeta_{0}{ }^{\prime}+r^{2} B_{0}^{\prime} \zeta_{0}-2 r B_{0} \zeta_{0}}{2\left[2 r^{2} B_{0}\right] \zeta_{0}}=-\frac{2 \zeta_{0}{ }^{\prime}}{\zeta_{0}}-\frac{B_{0}^{\prime}}{2 B_{0}}+\frac{1}{r} .
$$

Integrating over $r$,

$$
\int \frac{Z^{\prime \prime}{ }_{0}}{Z_{0}^{\prime}} d r=-\int \frac{2 \zeta_{0}{ }^{\prime}}{\zeta_{0}} d r-\int \frac{B_{0}^{\prime}}{2 B_{0}} d r+\int \frac{1}{r} d r,
$$

we obtain

$$
\ln Z_{0}^{\prime}=-2 \ln \zeta_{0}-\frac{1}{2} \ln B_{0}+\ln r+c
$$

Then,

$$
Z_{0}^{\prime}=\epsilon \frac{r}{\zeta_{0}^{2} \sqrt{B_{0}}}
$$

Finally,

$$
Z_{0}(r)=\sigma+\epsilon \int \frac{r d r}{\zeta_{0}(r)^{2} \sqrt{B_{0}(r)}}
$$

Proof for idempotent property. For a second application of theorem 2, the mapping is

$$
T_{2}\left(\sigma_{2}, \epsilon_{2}\right) \circ T_{2}\left(\sigma_{1}, \epsilon_{1}\right):\left\{\zeta_{0}, B_{0}\right\} \mapsto\left\{\zeta_{2}, B_{0}\right\},
$$

where $\zeta_{2}=\zeta_{1} Z_{1}\left(\zeta_{1}, B_{0}\right), \zeta_{1}=\zeta_{0} Z_{0}\left(\zeta_{0}, B_{0}\right)$ and $d Z_{0}=$ $\epsilon_{1} \frac{r d r}{\zeta_{0}^{2} \sqrt{B_{0}}}$. So,

$$
\begin{gathered}
\zeta_{1}=\zeta_{0} Z_{0}\left(\zeta_{0}, B_{0}\right) \\
\zeta_{2}=\zeta_{0} Z_{0}\left(\zeta_{0}, B_{0}\right) Z_{1}\left(\zeta_{1}, B_{0}\right) \\
=\zeta_{0} Z_{0}\left(\sigma_{2}+\epsilon_{2} \int \frac{r d r}{\zeta_{1}^{2} \sqrt{B_{0}(r)}}\right) \\
=\zeta_{0} Z_{0}\left(\sigma_{2}+\frac{\epsilon_{2}}{\epsilon_{1}} \int \frac{r d r}{\left(\zeta_{0} Z_{0}\right)^{2} \sqrt{B_{0}(r)}}\right) \\
=\zeta_{0} Z_{0}\left(\sigma_{2}+\frac{\epsilon_{2}}{\epsilon_{1}} \int \frac{d Z_{0}}{Z_{0}^{2}}\right) \\
=\zeta_{0} Z_{0}\left(\sigma_{2}-\frac{\epsilon_{2}}{\epsilon_{1}}\left[\frac{1}{Z_{0}}-\frac{1}{\sigma_{1}}\right]\right) \\
=\zeta_{0}\left(-\frac{\epsilon_{2}}{\epsilon_{1}}+Z_{0}\left[\sigma_{2}+\frac{\epsilon_{2}}{\epsilon_{1}} \frac{1}{\sigma_{1}}\right]\right)
\end{gathered}
$$

then

$$
Z_{1}=-\frac{\epsilon_{2}}{\epsilon_{1}}+Z_{0}\left[\sigma_{2}+\frac{\epsilon_{2}}{\epsilon_{1}} \frac{1}{\sigma_{1}}\right]
$$

As above, the composition law for theorem 1 is

$$
T_{2}\left(\sigma_{2}, \epsilon_{2}\right) \circ T_{2}\left(\sigma_{1}, \epsilon_{1}\right)=T_{2}\left(\sigma_{2} \sigma_{1}, \epsilon_{1} \sigma_{2}+\frac{\epsilon_{2}}{\sigma_{1}}\right) .
$$

Theorem 2 has idempotent property only in special case $\left(\sigma=1\right.$ for all $\sigma_{n}$ )

$$
\prod_{i=1}^{n} T_{2}\left(1, \epsilon_{i}\right)=T_{2}\left(1, \sum_{i=1}^{n} \epsilon_{i}\right),
$$

and for $\mathrm{n}$ application of theorem 2 with the same $\sigma$ and $\epsilon$

$$
\begin{gathered}
T_{2}(\sigma, \epsilon)^{n}=T_{2}\left(\sigma^{n}, \epsilon\left[\sigma^{n-1}+\sigma^{n-3}+\cdots\right.\right. \\
\left.\left.+\sigma^{-(n-1)}+\sigma^{-(n-3)}\right]\right) .
\end{gathered}
$$

For large $n$

$$
T_{2}(\sigma, \epsilon)^{n} \approx T_{2}\left(\sigma^{n}, \epsilon^{n}\right)=\sigma^{n-1} T_{2}(\sigma, \epsilon) \triangleq T_{2}(\sigma, \epsilon) .
$$

Corollary 2. Let $\left\{\zeta_{0}, B_{a}\right\}$ and $\left\{\zeta_{0}, B_{b}\right\}$ both represent charged fluid spheres, then for all $\mathrm{p}$

$$
\left\{p \zeta_{a}+q \zeta_{b}, B_{0}\right\}
$$

is also a charged fluid sphere. Furthermore, all charged fluid spheres for a fixed $B_{0}$ can also be written in this form.

Proof for corollary 2. Suppose $\left\{\zeta_{a}, B_{0}\right\}$ and $\left\{\zeta_{b}, B_{0}\right\}$ represent charged fluid spheres, the solutions could satisfy (7)

$$
\begin{gathered}
2 r^{2} B_{0}\left(\zeta_{a}\right)^{\prime \prime}+\left(r^{2} B_{0}{ }^{\prime}-2 B_{0} r\right)\left(\zeta_{a}\right)^{\prime} \\
+\left(2-2 B_{0}+B_{0}{ }^{\prime} r+16 \pi \Delta r^{2}\right)\left(\zeta_{a}\right)=0,
\end{gathered}
$$

and

$$
\begin{gathered}
2 r^{2} B_{0}\left(\zeta_{b}\right)^{\prime \prime}+\left(r^{2} B_{0}{ }^{\prime}-2 B_{0} r\right)\left(\zeta_{b}\right)^{\prime} \\
+\left(2-2 B_{0}+B_{0}{ }^{\prime} r+16 \pi \Delta r^{2}\right)\left(\zeta_{b}\right)=0 .
\end{gathered}
$$

$\left\{p \zeta_{a}+q \zeta_{b}, B_{0}\right\}$ also represents charged fluid spheres,

$$
\begin{aligned}
& 2 r^{2} B_{0}\left(p \zeta_{a}+q \zeta_{b}\right)^{\prime \prime}+\left(r^{2} B_{0}{ }^{\prime}-2 B_{0} r\right)\left(p \zeta_{a}+q \zeta_{b}\right)^{\prime} \\
& +\left(2-2 B_{0}+B_{0}{ }^{\prime} r+16 \pi \Delta r^{2}\right)\left(p \zeta_{a}+q \zeta_{b}\right)=0 \\
& 2 r^{2} B_{0}\left(p \zeta_{a}^{\prime \prime}+q \zeta_{b}^{\prime \prime}\right)+\left(r^{2} B_{0}{ }^{\prime}-2 B_{0} r\right)\left(p \zeta_{a}{ }^{\prime}+q \zeta_{b}{ }^{\prime}\right) \\
& +\left(2-2 B_{0}+B_{0}{ }^{\prime} r+16 \pi \Delta r^{2}\right)\left(p \zeta_{a}+q \zeta_{b}\right)=0 .
\end{aligned}
$$

We found $(35)=[p *(33)+(q) *(34)]$. Therefore, $\left\{p \zeta_{a}+q \zeta_{b}, B_{0}\right\}$ represent a charged fluid sphere.

Application of theorem 2 to the Reissner-Nordstrom metric

\section{Starting metric:}

\section{Reissner-NordstrÖm}

$\mathrm{T}_{2}$

\section{New solution: equation (36.b)}

Figure 3. This structure shows that when we apply theorem 2 to the Reissner-Nordstrom metric, we get a new solution.

The given form of the Reissner-Nordstrom metric is $d s^{2}=-\left(1-\frac{R_{s}}{r}+\frac{R_{Q}{ }^{2}}{r^{2}}\right) d t^{2}+\left(1-\frac{R_{s}}{r}+\frac{R_{Q}{ }^{2}}{r^{2}}\right)^{-1} d r^{2}$, 
then

$$
\zeta_{0}=\sqrt{1-\frac{R_{s}}{r}+\frac{R_{Q}^{2}}{r^{2}}}
$$

and

$$
B_{0}=1-\frac{R_{S}}{r}+\frac{R_{Q}^{2}}{r^{2}}
$$

Applying theorem 2, use equation (24)

$$
\begin{gathered}
Z_{0}(r)=\sigma+\epsilon \int \frac{r d r}{\zeta_{0}(r)^{2} \sqrt{B_{0}(r)}}, \\
Z_{0}(r)=\sigma+\epsilon \int \frac{r d r}{\left(1-\frac{R_{S}}{r}+\frac{R_{Q}^{2}}{r^{2}}\right)^{3 / 2}},
\end{gathered}
$$

From calculations using the Maple programme, we obtain

$$
Z_{0}=\sigma+\epsilon\left[\left(\frac{15}{8} R_{s}{ }^{2}-\frac{3}{2} R_{Q}{ }^{2}\right) \ln \left(-\frac{1}{2} R_{s}+r+I\right)\right.
$$

$$
\begin{gathered}
+\frac{1}{I}\left(\frac{1}{2} r^{3}+\frac{5}{4} R_{s} r^{2}+\left(\frac{3}{2} B^{2}-\frac{15}{8} R_{s}{ }^{2}\right) r\right) \\
\frac{1}{\left(-R_{s}{ }^{2}+4 R_{Q}{ }^{2}\right) I}\left(\left(\frac{15}{8} R_{s}{ }^{4}-\frac{13}{2} R_{Q}{ }^{2} R_{s}{ }^{2}\right) r\right. \\
\left.\left.+\left(\frac{13}{4} R_{Q}{ }^{2} R_{s}{ }^{3}-\frac{15}{16} R_{s}{ }^{5}\right)\right)\right],
\end{gathered}
$$

where

$$
I=\sqrt{-R_{S} r+R_{Q}^{2}+r^{2}},
$$

then for all $\sigma$ and $\epsilon$, the new metric is

$$
d s^{2}=-\zeta_{0}(r)^{2} Z_{0}^{2} d t^{2}+\frac{d r^{2}}{B_{0}(r)}+r^{2} d \Omega^{2} .
$$

With this $Z_{0}$, the metric also satisfy anisotropy fluid spheres.

Example of some starting metrics

Table 1. This table shows the structures of charged anisotropy metric [11-14].

\begin{tabular}{ll}
\hline Name & Metric form $\left(\boldsymbol{d s}^{2}\right)$ \\
\hline Reissner-Nordstrom (for a non-ratating charged spherical object) & $\left(1-\frac{r_{s}}{r}+\frac{r_{Q}{ }^{2}}{r^{2}}\right) c^{2} d t^{2}-\left(1-\frac{r_{s}}{r}+\frac{r_{Q}{ }^{2}}{r^{2}}\right)^{-1} d r^{2}-r^{2} d \Omega^{2}$ \\
Tikekar (for positive pressure and negative gradient pressure) & {$\left[A\left(-\frac{11}{24}-\frac{7 r^{2}}{12 R^{2}}+\frac{49 r^{4}}{24 R^{4}}\right)+B \sqrt{\left.1-\frac{r^{2}}{R^{2}}\left(\frac{1}{8}+\frac{7 r^{2}}{8 R^{2}}\right)^{3 / 2}\right]^{2} d t^{2}-\frac{1}{B_{0}} d r^{2}-r^{2} d \Omega^{2}}\right.$} \\
$\begin{array}{l}\text { Tolman-Bayin (specific choice } \mathrm{n}=0 \text { and the total gravitational } \\
\text { mass, } \mathrm{m}(\mathrm{r}=0) \text { ) }\end{array}$ & $\left(1-\frac{2 m}{a}+\frac{q^{2}}{a^{2}}\right)^{2} d t^{2}-\left(1-\frac{2 q^{2}}{a^{2}}\right)^{-2} d r^{2}-r^{2} d \Omega^{2}$ \\
$\begin{array}{l}\text { Psuedo-spheroidal (where A and B are constants, and } u^{2}= \\
\{k /(k-1)\}\left(1+r^{2} / R^{2}\right)\end{array}$ & $\left(A u+B\left\{u \log \left(u+\sqrt{u^{2}-1}\right)-\sqrt{u^{2}-1}\right\}\right)^{2} d t^{2}-\frac{1+\frac{k r^{2}}{R^{2}}}{1+\frac{r^{2}}{R^{2}}} d r^{2}-r^{2} d \Omega^{2}$ \\
\hline
\end{tabular}

\subsubsection{Classifying Charged Fluid Spheres}

In referrence to P. Boonserm et. al. [2], we use a similar concept to classify charged fluid spheres into seed and non-seed metrics based on the following definitions.

Definition (seed metric): take a metric $g$ and apply theorem 1 or theorem 2 to it. Two different cases are possible $[2,10,15]$ : each of the applications supplies us with a new solution, $\left[T_{1}(g) \triangleq g \triangleq T_{2}(g)\right]$. We define a metric with this pattern as a seed metric. For example, figure 2 shows that when we apply theorem 1 to the Reissner-Nordstrom metric, we derive a new solution (see equation 23). In addition, figure 3 shows that when we apply theorem 2 to the Reissner-Nordstrom metric, we derive a new solution (see equation 39).

Definition (non-seed metric): take a metric $g$ and apply theorem 1 or theorem 2 to it. Two different cases are possible: only one of the applications supplies us with a new solution, while the other one gives us the same metric we started with $\left[T_{1}(g) \triangleq g\right]$ or $\left[T_{2}(g) \triangleq g\right]$. These metrics are non-seed metrics.

In the classifying step, the generated solutions are classified either as seed or non-seed solutions. If the generated solution can give rise to a new solution after reapplying it with every generating theorem, the solution is regarded as a seed type. On the other hand, if the reapplication of the generated solution with the generating theorems only results in a new solution from just one generating theorem, the solution is classified as a non-seed type. We also created a relationship among the two theorems.

\section{Conclusion}

The solution generating theorems for anisotropy can be constructed in Schwarzschild coordinate. The theorems can generate solutions that satisfy anisotropy. The idempotent property and corollary of the theorems were presented in this work. The application of the solution generating theorems has been tested with the Reissner-Nordstrom metric and has been shown to generate solutions. Finally, we can classify the types of solutions into seed and non-seed, and we can find the interrelationship between the solutions.

\section{Acknowledgements}

This project was funded by the Ratchadapisek Sompoch Endowment Fund, Chulalongkorn University (Sci-Super 
2014-032), by a grant for the professional development of new academic staff from the Ratchadapisek Somphot Fund at Chulalongkorn University, by the Thailand Research Fund (TRF), and by the Office of the Higher Education Commission (OHEC), Faculty of Science, Chulalongkorn University. PB was additionally supported by a scholarship from the Royal Government of Thailand. TN was also additionally supported by a scholarship from the Development and Promotion of Science and Technology talent project (DPST). This work is supported by CUniverse research promotion project by Chulalongkorn University (grant reference CUAASC).

\section{References}

[1] K. Schwarzschild, "On the gravitational field of a sphere of incompressible fluid according to Einstein's theory", Sitzungsber. Preuss. Akad. Wiss. Berlin (Math. Phys.) 1916 (1916) 424 [arXiv: physics/9912033 [physics. hist-ph]].

[2] P. Boonserm, M. Visser and S. Weinfurtner, "Generating perfect fluid spheres in general relativity", Phys. Rev. D 71 (2005) 124037 [arXiv: gr-qc/0503007].

[3] S. Carroll, "Spacetime and geometry: an introduction to general relativity", Pearson new international edition, U.S.A., Pearson Education Limited, 2014.

[4] H. Bondi, "Spherically symmetrical models in general relativity”, Mon. Not. Roy. Astron. Soc. 107 (1947) 410.

[5] H. A. Buchdahl, "General relativistic fluid spheres", Phys. Rev. 116 (1959) 1027-1034.

[6] M. S. R. Delgaty and K. Lake, "Physical acceptability of isolated, static, spherically symmetric, perfect fluid solutions of Einstein's equations", Compute. Phys. Commun. 115 (1998) 395 [arXiv: gr-qc/9809013]

[7] P. Boonserm, M. Visser and S. Weinfurtner, "Solution generating theorems for the TOV equation", Phys. Rev. D 76 (2007) 044024 [arXiv: gr-qc/0607001].
[8] A. Sulaksono, "Anisotropic pressure and hyperon in neutron stars,” Int. J. Mod. Phys. E 24 (2015) 01, 1550007 [arXiv: 1412.7274 [nucl-tn]].

[9] P. Boonserm, T. Ngampitipan and M. Visser, "Mimicking static anisotropic fluid spheres in general relativity", International Journal of Modern Physics D (2015): 1650019 [arXiv: $1501.07044 \mathrm{v} 3$ [gr-qc]].

[10] P. Boonserm, "Some exact solution in general relativity", MSc. Thesis, Victoria University of Wellington, 2006.

[11] Chandrasekhar, S. (1998). The Mathematical Theory of Black Holes (Reprinted ed.). Oxford University Press. p. 205. ISBN 0-19850370-9. Retrieved 13 May 2013.

[12] K. Komathiraj ans S. D. Maharaj, "A class of charged relativistic spheres", Mathematical and Computational Applications 15, 665-673, 2010.

[13] Patel, L. K., and N. P. Mehta. "An exact model of an anisotropic relativistic sphere." Australian Journal of Physics 48.4 (1995): 635-644.

[14] Ray, Saibal, and Basanti Das, "Tolman-Bayin type static charged fluid spheres in general relativity." Monthly Notices of the Royal Astronomical Society 349.4 (2004), 1331-1334.

[15] K. Thairatana, "Transformation for perfect fluid spheres in isotropic coordinates", MSc. Thesis, Chulalongkorn University, 2013.

[16] K. Lake, "all static spherically symmetric perfect fluid solutions of Einstein's equation", Phys. Rev. D 67 (2003) 104015 [gr-qc/0209104].

[17] S. Rahman and M. Visser, "Space-time geometry of static fluid spheres", Class. Quant. Grav. 19 (2002) 935 [gr-qc/0103065].

[18] S. S. Bayin, "Anisotropic fluid spheres in general relativity", Phys. Rev. D 26 (1982) 1262.

[19] L. Herrera, J. Ospino and A. Di Prisco, "All static spherically symmetric anisotropic solutions of Einstein's equation", Phys. Rev. D 77 (2008) 027502 [arXiv: 0712.0713 [gr-qc]]. 